\title{
Piston Resonance in the Orifice Pulse Tube (preliminary draft)
}

\author{
P.C.T. de Boer, ${ }^{1}$ J.-M. Duval, I. Charles and L. Duband \\ Département de Recherche Fondamentale sur la Matière Condensée \\ Service des Basses Températures, CEA-Grenoble \\ 17 rue des Martyrs, 38054 Grenoble Cédex 9, France
}

\begin{abstract}
The force exerted on the piston of a linear motor driving an orifice pulse tube is analyzed using linearized theory. First, the case of a pulse tube without orifice and reservoir is considered. It is found that there are two possible resonances, corresponding to a very small and a very large orifice conductance, respectively. In principle, such resonances can be used to reduce the current provided to the linear motor, and hence to reduce the associated ohmic losses. There is no resonance of the power provided to the pulse tube. An expression is derived for the dissipation in the tube chambers associated with heat losses to the wall. Experimental results obtained for the phase angle and the amplitude of the piston force indicate that there is a large influence of turbulent effects on thermal diffusivity. The analysis is extended to the case with regenerator. It is found that dissipation as well large temperature ratios across the regenerator tend to decrease the piston resonance effect.
\end{abstract}

\section{NOMENCLATURE}

\begin{tabular}{llll}
$A$ & cross sectional area & \multicolumn{2}{l}{ Greek symbols } \\
$C$ & conductance & $\bar{\alpha}$ & thermal diffusivity, $k /\left(\rho c_{p}\right)$ \\
$C_{A}$ & $C_{\epsilon} D /\left(2 y^{2}\right)$ & $\Delta$ & denotes amplitude \\
$C_{\epsilon}$ & see Eq. $(2)$ & $\delta$ & phase angle \\
$c$ & specific heat & $\gamma$ & ratio of specific heats \\
$C O_{o}$ & $C_{o} / C_{r}$ & $\lambda$ & characteristic thermal boundary layer \\
$C O_{r}$ & see Eq. $(39)$ & & thickness, $(2 \bar{\alpha} / \omega)^{1 / 2}$ \\
$D$ & distance between parallel plates & $\pi_{b}$ & $\Delta p_{b} / \Delta p_{p}$ \\
$F$ & force & $\pi_{p}$ & $\Delta p_{p} / \Delta p_{d}$ \\
$G$ & $(\gamma-1) S D /(4 V)$ & $\rho$ & density \\
$\dot{m}$ & mass flow rate & $\Omega$ & frequency, see Eqs.16, 22 and 36
\end{tabular}

\footnotetext{
${ }^{1}$ Present address: Cornell University, Ithaca, NY 14850. Tel. (607) 255-3583, Fax (607)255-3583. E-mail PTD1@cornell.edu.
} 


\begin{tabular}{llll}
$P_{o}$ & average pressure & $\omega$ & \multicolumn{2}{c}{ angular frequency } \\
$p$ & pressure & \multicolumn{2}{c}{ Subscripts } \\
$\dot{Q}$ & rate of heat flow into system & $b$ & buffer (reservoir) \\
$\dot{q}$ & heat flux into wall & $c$ & cold \\
$S$ & surface area & $d$ & driver \\
$s$ & $2 x /(D)$ & $h$ & hot \\
$T$ & temperature & $o$ & orifice; average \\
$t$ & time & $p$ & pulse tube \\
$U$ & internal energy & $r$ & regenerator \\
$V$ & volume & $w$ & wall \\
$\dot{W}$ & rate of work done by system & & \\
$x$ & distance from centerplane & & \\
$y$ & $(D) /(2 \lambda)$ & & \\
$z$ & $(1+i) y$ & &
\end{tabular}

\section{INTRODUCTION}

In the last decade or so, the pulse tube has been developed as an efficient and reliable cryocooler [1]. Many important improvements have been made. These include the introduction of an inertance tube rather than an orifice to provide the required phase difference between mass flow rate and pressure $[2,3]$. The inertance tube is useful only for pulse tubes operating at relatively high frequencies. For pulse tubes operating at relatively low frequencies, a major improvement has been the introduction of a second orifice in a tube that short-circuits the regenerator [4]. Current efforts toward further improvements mostly concern efforts to decrease various losses inherent in the operation of the tube.

A potential improvement in the operation of pulse tubes driven by linear motors is the reduction of ohmic losses associated with the electric current provided to the motor. The piston driven by the motor can be at resonance with the pressure pulsations in the drive chamber. The resonance corresponds to a minimum of the amplitude of the force exerted by the linear motor to the piston. This force is proportional to the current passing through the motor. Operating the pulse tube at piston resonance thus would reduce ohmic losses. Operation near resonant frequency is especially critical for large machines [5]

The value of the amplitude of the force at resonance is limited by the occurrence of dissipation in the drive chamber and pulse tube, caused by irreversible heat transfer to the walls. Mirels [6] used the linearized result presented by Lee [7] for the rate of this heat transfer. This result is based on assuming molecular diffusivity in the thermal boundary layer as well as in the region outside the boundary layer. The heat transfer is a second order effect, which arises from the phase difference which exists between pressure and first order heat transfer. Theoretical results of this model are in satisfactory agreement with experimental data for a single gas spring without high velocity inflows [8]. Later work carried out in connection with Stirling engine research by Cantelmi et al. [9] showed that in actual engine cylinders, inflow generated turbulence can significantly increase the rate of heat transfer to the walls. To account for this effect, Cantelmi et al. [9] used a turbulence enhanced thermal diffusivity.

The present work begins with extending the treatment of [9] from the case of a single wall in contact with a semi-infinite gas to the case of two parallel walls. This extension is of importance in the case of large enhancement of thermal diffusivity by turbulence. Next, the theory presented by Mirels [6] is extended to a pulse tube with heat losses in the reservoir and the pulse tube, as well as in the drive chamber. Expressions are developed for the phase angle and the amplitude of the force exerted on the piston. It is found that piston resonance can occur both at small and at large values of the dimensionless conductance of the regenerator. The power delivered by the piston to the gas does not exhibit a resonance. The expressions 
derived for the phase angle and force are compared with experimental results. The latter were obtained in an set-up similar to that of a pulse tube, but with the regenerator replaced by a valve of variable conductance, and without an orifice and reservoir. The experimental heat losses to the wall are found to correspond with a large enhancement of diffusivity by turbulent effects. As a result of this enhancement, the resonance effect can be significantly reduced. This is shown for a complete pulse tube in the last section of the paper. It is found that the temperature difference across the regenerator also tends to reduce the resonance effect significantly.

Following the analyses of the works cited, the present treatment uses complex notation. Physical quantities are given by the real parts of the corresponding parameters.

\section{RATE OF HEAT TRANSFER TO THE WALL}

The rate at which heat is transferred from the gas to the wall can be estimated using the method described by Lee [7] and extended by Mirels [6] and Cantelmi et al. [9]. The method is based on using the simplified energy equation

$$
\frac{\partial T}{\partial t}=\bar{\alpha} \frac{\partial}{\partial x}\left(1+\frac{\epsilon_{H}}{\bar{\alpha}}\right) \frac{\partial T}{\partial x}+\frac{1}{\rho c_{p}} \frac{\partial p}{\partial t} .
$$

The quantity $\epsilon_{H} / \bar{\alpha}$ represents the ratio of the turbulent eddy diffusivity to the molecular diffusivity. Cantelmi et al. [9] considered the case of a single plate in thermal contact with a semi-infinite gas. They took $\epsilon_{H} / \bar{\alpha}$ to be proportional to the distance from the wall. Here, Eq. (1) is applied to the case of two infinite parallel plates. The ratio $\epsilon_{H} / \bar{\alpha}$ is taken to be proportional to the distance to the closest wall

$$
\epsilon_{H} / \bar{\alpha}=C_{\epsilon}(D / 2-|x|)
$$

The solution of Eq. (1) under the boundary conditions $T=T_{o}$ at $x= \pm D / 2$ and $\partial T / \partial x=0$ at $x=0$ is

$$
T(x, t)=T_{o}+\frac{\Delta p}{\rho c_{p}}\left[1-\frac{K_{o}(\zeta)+I_{o}(\zeta) K_{1}(\theta) / I_{1}(\theta)}{K_{o}\left(\zeta_{w}\right)+I_{o}\left(\zeta_{w}\right) K_{1}(\theta) / I_{1}(\theta)}\right] e^{i \omega t}
$$

where $\zeta=\theta \sqrt{1-C_{\epsilon}^{\prime} x}, \zeta_{w}=\theta \sqrt{1-C_{\epsilon}^{\prime} D / 2}, C_{\epsilon}^{\prime}=C_{\epsilon} /\left(1+C_{\epsilon} D / 2\right), \theta=2(1+i) /\left(C_{A} y^{2}\right)$, $C_{A}=C_{\epsilon} D /\left(2 y^{2}\right)$, and where $K$ and $I$ are the modified Bessel functions. Cantelmi et al. [9] took $C_{A}$ to be proportional to the ratio of the cross section $A_{c y l}$ of the cylinder to the cross section $A_{\text {inlet }}$ of the inlet tube. They noted that the value of $C_{A} y=C_{\epsilon} \lambda$ is a measure of the magnitude of the eddy diffusivity in the thermal boundary layer.

The heat flux to the wall is given by

$$
\dot{q}=-k\left[\frac{\partial T}{\partial(s D / 2)}\right]_{s=0}=\frac{D}{4} \omega \Delta p e^{i \omega t}(a+b i)
$$

where

$$
a+b i=\frac{z}{y^{2}} \frac{K_{1}\left(\zeta_{w}\right)-I_{1}\left(\zeta_{w}\right) K_{1}(\theta) / I_{1}(\theta)}{K_{o}\left(\zeta_{w}\right)+I_{o}\left(z e t a_{w}\right) K_{1}(\theta) / I_{1}(\theta)}
$$

\section{ANALYSIS OF ORIFICE PULSE TUBE}

The model on which this analysis is based is sketched in Fig.1. The analysis is an extension of that presented by Mirels [6]. The three chambers are analyzed sequentially. 


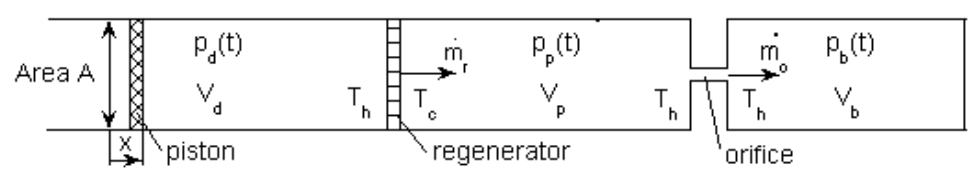

Figure 1: Sketch of model used

Each of them is subject to the time-dependent first law of thermodynamics for a control volume with at most one inlet $(i)$ and one exit $(e)$

$$
\frac{d U}{d t}=\dot{m}_{i} h_{i}-\dot{m}_{e} h_{e}+\dot{Q}-\dot{W}
$$

Here,

$$
U=\int_{V} c_{v} T \rho d V=\frac{p V}{\gamma-1}
$$

where use was made of the ideal gas law. The pressures and mass flow rates are written as follows

$$
\begin{array}{r}
p_{b}(t)=\Delta p_{b} e^{i\left(\omega t+\delta_{b}\right)}+P_{o} \\
p_{p}(t)=\Delta p_{p} e^{i \omega t}+P_{o} \\
p_{d}(t)=\Delta p_{d} e^{i\left(\omega t+\delta_{d}\right)}+P_{o} \\
\dot{m}_{o}=\rho_{h} C_{o}\left[p_{p}(t)-p_{b}(t)\right] \\
\dot{m}_{r}=\rho_{c} C_{r}\left[p_{d}(t)-p_{p}(t)\right]
\end{array}
$$

Application of Eq. (6) to the reservoir ("buffer") involves setting $p=p_{b}(t), V=V_{b}=$ constant, $\dot{m}_{i}=\dot{m}_{o}, h_{i}=c_{p} T_{h}, \dot{m}_{e}=0, \dot{W}=0$ and $\dot{Q}=-\dot{q}_{b} S_{b}$. This yields, after some algebra

$$
\pi_{b} e^{i \delta_{b}}\left(\alpha_{b}-\beta_{b} i\right)=-i / \Omega_{b}
$$

where

$$
\begin{array}{r}
\alpha_{b}=1+G_{b} b_{b}, \\
\beta_{b}=\Omega_{b}^{-1}+G_{b} a_{b} \\
\Omega_{b}=\frac{\omega V_{b}}{\gamma P_{o} C_{o}}, \quad \pi_{b}=\frac{\Delta p_{b}}{\Delta p_{p}}
\end{array}
$$

It follows that

$$
\begin{gathered}
\pi_{b}=\frac{1}{\Omega_{b} \sqrt{\alpha_{b}^{2}+\beta_{b}^{2}}} \\
\tan \left(\delta_{b}\right)=-\alpha_{b} / \beta_{b}
\end{gathered}
$$

Next, Eq.6 is applied to the pulse tube. Noting that now $p=p_{p}(t), V=V_{p}=$ constant, $\dot{m}_{i}=\dot{m}_{r}, h_{i}=c_{p} T_{c}, \dot{m}_{e}=\dot{m}_{o}, h_{e}=c_{p} T_{h}, \dot{W}=0$ and $\dot{Q}=-\dot{q}_{p} S_{p}$, this yields

$$
\pi_{p} e^{-i \delta_{d}}\left(\alpha_{p}-\beta_{p} i\right)=-i \Omega_{p}^{-1}
$$

where

$$
\begin{array}{r}
\alpha_{p}=1+\frac{V_{b}}{V_{p}} \frac{1}{\Omega_{b}^{2}} \frac{\alpha_{b}}{\alpha_{b}^{2}+\beta_{b}^{2}}+G_{p} b_{p}, \\
\beta_{p}=\frac{1}{\Omega_{p}}+\frac{V_{b}}{V_{p}} \frac{1}{\Omega_{b}^{2}}\left(\Omega_{b}-\frac{\beta_{b}}{\alpha_{b}^{2}+\beta_{b}^{2}}\right)+G_{p} a_{p}, \\
\Omega_{p}=\frac{\omega V_{p}}{\gamma P_{o} C_{r}}, \quad \pi_{p}=\frac{\Delta p_{p}}{\Delta p_{d}}
\end{array}
$$


It follows that

$$
\begin{array}{r}
\pi_{p}=\frac{1}{\Omega_{p} \sqrt{\alpha_{p}^{2}+\beta_{p}^{2}}} \\
\tan \left(\delta_{d}\right)=\alpha_{p} / \beta_{p} .
\end{array}
$$

Application of Eq. (6) to the driver section requires setting $\left.p=p_{d}(t), V=V_{d}-A x(t)\right)$, $\dot{m}_{i}=0, \dot{m}_{e}=\dot{m}_{r}, h_{e}=c_{p} T_{h}, \dot{W}=-P_{o} A d x / d t=P_{o} d V_{d} / d t, \dot{Q}=-\dot{q}_{d} S_{d}$. Setting furthermore

$$
x(t)=\Delta x e^{i\left(\omega t+\delta_{x}\right)}
$$

it is found that

$$
\frac{\gamma P_{o} A \Delta x e^{i \delta_{x}}}{V_{d} \Delta p_{d} e^{i \delta_{d}}}=\alpha_{d}-\beta_{d} i
$$

where

$$
\begin{array}{r}
\alpha_{d}=1+\frac{T_{h}}{T_{c}} \frac{V_{p}}{V_{d}} \frac{1}{\Omega_{p}^{2}} \frac{\alpha_{p}}{\alpha_{p}^{2}+\beta_{p}^{2}}+G_{d} b_{d}, \\
\beta_{d}=\frac{T_{h}}{T_{c}} \frac{V_{p}}{V_{d}} \frac{1}{\Omega_{p}^{2}}\left(\Omega_{p}-\frac{\beta_{p}}{\alpha_{p}^{2}+\beta_{p}^{2}}\right)+G_{d} a_{d} .
\end{array}
$$

It follows that

$$
\begin{gathered}
\frac{\gamma P_{o} A \Delta x}{V_{d} \Delta p_{d}}=\sqrt{\alpha_{d}^{2}+\beta_{d}^{2}}, \\
\tan \left(\delta_{x}-\delta_{d}\right)=-\beta_{d} / \alpha_{d} .
\end{gathered}
$$

Taking $\gamma=5 / 3$, the value of $G \equiv(\gamma-1) S D /(4 V)$ in the foregoing results is $1 / 3$ for 2 infinite plates. Provided $y \gg 1$, the results may be applied to other geometries by substituting the actual value of the surrounding surface for $S$, and the value of the actual volume for $V$ [6]. For a cylinder this yields $G=2 / 3$, for a sphere $G=1$.

\section{FORCE ON PISTON}

The force on the piston is found from the equation

$$
\tilde{F} \equiv \Delta \tilde{F} e^{i\left(\omega t+\delta_{F}\right)}=m \frac{d^{2} \Delta x e^{i\left(\omega t+\delta_{x}\right)}}{d t^{2}}+A \Delta p_{d} e^{i\left(\omega t+\delta_{d}\right)} .
$$

Working this out yields

$$
\frac{\Delta F e^{i \delta_{F}}}{\Delta x e^{i \delta_{x}}}=\frac{\gamma P_{o} A^{2}}{V_{d}}\left(-\Omega_{m}^{2}+\frac{\alpha_{d}+\beta_{d} i}{\alpha_{d}^{2}+\beta_{d}^{2}}\right),
$$

from which

$$
\begin{array}{r}
\Delta \tilde{F}=\frac{\gamma P_{o} A^{2} \Delta x}{V_{d}} \Delta F, \\
\tan \left(\delta_{F}-\delta_{x}\right)=\frac{\beta_{d}}{\alpha_{d}-\Omega_{m}^{2}\left(\alpha_{d}^{2}+\beta_{d}^{2}\right)},
\end{array}
$$

where use was made of Eq. (26), and where

$$
\begin{array}{r}
\Delta F=\left(\Omega_{m}^{4}-2 \Omega_{m}^{2} \frac{\alpha_{d}}{\alpha_{d}^{2}+\beta_{d}^{2}}+\frac{1}{\alpha_{d}^{2}+\beta_{d}^{2}}\right)^{1 / 2}, \\
\Omega_{m}=\frac{\omega}{A} \sqrt{\frac{m V_{d}}{\gamma P_{o}}} .
\end{array}
$$




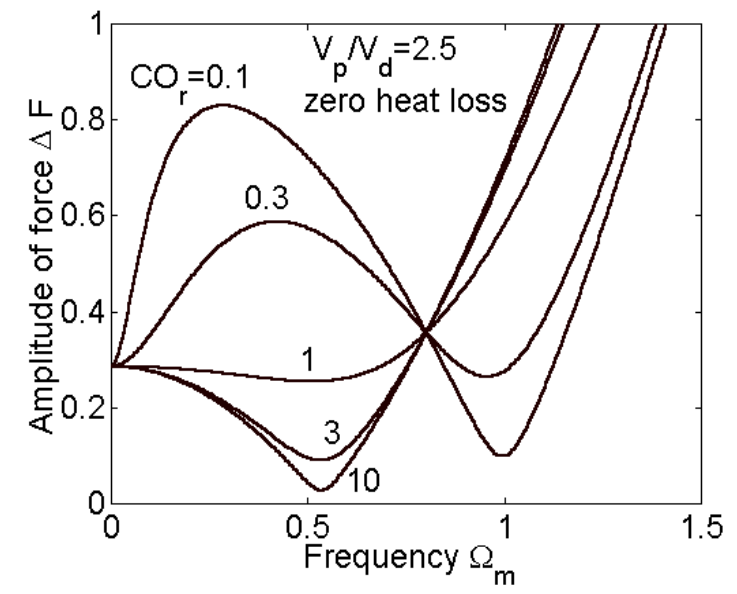

Figure 2: Amplitude $\Delta F$ of force as function of $\Omega_{m}$

\section{RESONANCES AT ZERO HEAT LOSS}

The resonance character of $\Delta F$ can be illustrated by considering the simplified case of zero heat loss. For the purposes of this illustration, the orifice is taken to be closed $\left(C_{o}=0\right)$, and the regenerator temperature is taken to be uniform $\left(T_{h} / T_{c}=1\right)$. For this case, $\Omega_{b}=\infty, a_{p}=b_{p}=a_{d}=b_{d}=0, \alpha_{p}=1, \beta_{p}=1 / \Omega_{p}, \alpha_{d}=1+\left(V_{p} / V_{d}\right) /\left(\Omega_{p}^{2}+1\right)$, $\beta_{d}=\left(V_{p} / V_{d}\right) \Omega_{p} /\left(\Omega_{p}^{2}+1\right)$. This leads to two possible resonances. One obtains in the limit of zero regenerator conductance $\left(C_{r}=0\right)$, which corresponds to a geometry consisting of the drive chamber, only. In this case, $\Omega_{p}=\infty, \alpha_{d}=1, \beta_{d}=0$, and

$$
\Delta F=\left|\Omega_{m}^{2}-1\right| \text {. }
$$

The second resonance obtains in the limit of infinitely large regenerator conductance $\left(C_{r}=\right.$ $\infty)$, which corresponds to a geometry consisting of a single chamber having the combined volume $V_{p}+V_{d}$ of the pulse tube and the drive chamber. Now $\Omega_{p}=0, \alpha_{d}=1+V_{p} / V_{d}$, $\beta_{d}=0$, and

$$
\Delta \tilde{F}=\frac{\gamma P_{o} A^{2} \Delta x}{V_{p}+V_{d}}\left|\Omega_{m p d}^{2}-1\right| .
$$

Here $\Omega_{m p d}=(\omega / A) \sqrt{\left[m V_{d} /\left(\gamma P_{o}\right)\right]}$ is the resonance frequency of the combined volume. The resonance at $\Omega_{m p d}=1$ corresponds to $\Omega_{m}=\sqrt{V_{d} /\left(V_{p}+V_{d}\right)}$. In considering intermediate value of $C_{r}$, the dimensionless conductance of the regenerator is defined as

$$
C O_{r} \equiv \frac{C_{r}}{A} \sqrt{\frac{\gamma m P_{o}}{V_{d}}}\left(=\frac{\Omega_{m}}{\Omega_{p}} \frac{V_{p}}{V_{d}}\right)
$$

Hence

$$
\Omega_{p}=\frac{\Omega_{m}}{C O_{r}} \frac{V_{p}}{V_{d}}
$$

Consequently, $\Delta F$ can be plotted as a function of $\Omega_{m}$ for given values of the parameters $C O_{r}$ and $V_{p} / V_{d}$. Such a plot is shown in Fig. 2 for $V_{p} / V_{d}=2.5$ and for various values of $C O_{r}$. The possible resonances at $\Omega_{m}=1$ and $\Omega_{m}=\sqrt{V_{d} /\left(V_{p}+V_{d}\right)}=0.535$ manifest themselves clearly. At values of $C O_{r}$ near unity, the value of $\Delta F$ is dominated by dissipation in the regenerator, and the resonance phenomenon disappears. Corresponding results for the phase angle $\delta_{F}-\delta_{x}$ are shown in Fig. 3. In the limiting cases $C O_{r}=0$ and $C O_{r}=\infty$, this phase angle jumps from 0 degrees just below resonance to 180 degrees just above. Its value at resonance is 90 degrees. 


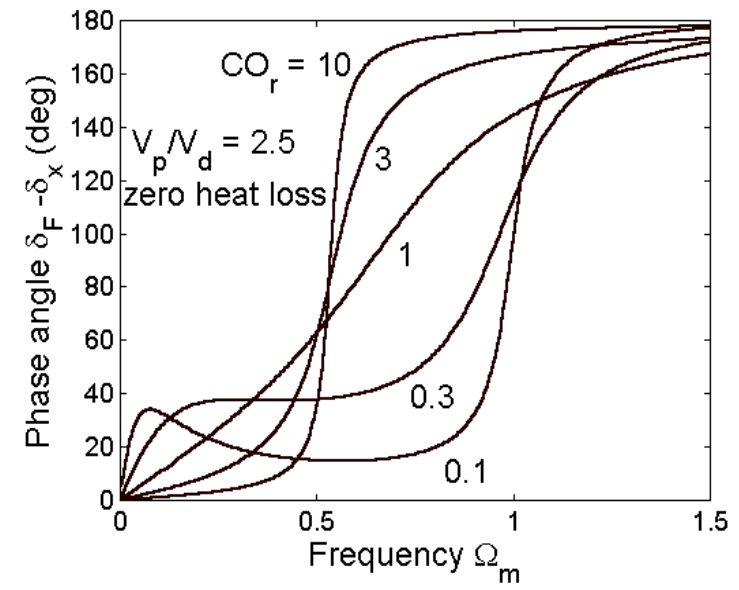

Figure 3: Phase angle $\delta_{F}-\delta_{x}$ as function of $\Omega_{m}$

\section{POWER DELIVERED BY PISTON}

The power delivered by the piston is given by

$$
\tilde{P}=\oint \tilde{F} \frac{d x}{d t} d t=\frac{\omega}{2 \pi} \int_{0}^{2 \pi / \omega} \Delta \tilde{F} \cos \left(\omega t+\delta_{F}\right) \frac{d}{d t} \Delta x \cos \left(\omega t+\delta_{x}\right) d t=\frac{\omega}{2} \Delta x \Delta \tilde{F} \sin \left(\delta_{F}-\delta_{x}\right) .
$$

Using Eq. (32) to substitute for $\Delta \tilde{F} \sin \left(\delta_{F}-\delta_{x}\right)$, there results

$$
\tilde{P}=\frac{\omega \gamma P_{o}(A \Delta x)^{2}}{2 V_{d}} P
$$

where the dimenensionless power $P$ equals

$$
P=\frac{\beta_{d}}{\alpha_{d}^{2}+\beta_{d}^{2}}
$$

This power $P$ is plotted in Fig. 4 as function of $\Omega_{m}$, for the values of $V_{p} / V_{d}$ and $C O_{r}$ also used in Figs. 2 and 3. It is seen that the power delivered by the piston to the gas does not exhibit minima corresponding to the minima in $\Delta F$ at $\Omega_{m}=1$ and $\Omega_{m p d}=1$. At these minima, the phase angle $\delta_{F}-\delta_{x}$ is 90 degrees (see Fig. 3). Consequently, the sine of this angle is at its maximum value. As a result, the product $\Delta \tilde{F} \sin \left(\delta_{F}-\delta_{x}\right)$ appearing in Eq. 41 for $\tilde{P}$ does not pass through an extremum.

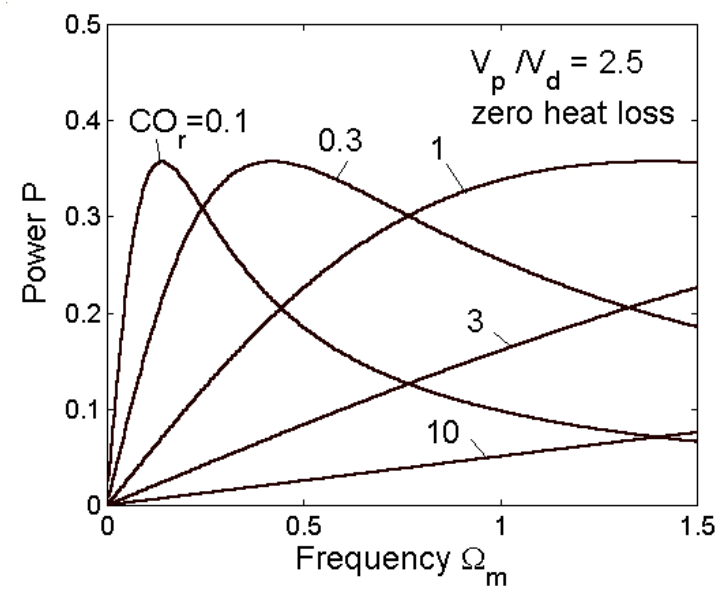

Figure 4: Dimensionless power $P$ as function of $\Omega_{m}$ 


\section{ESTIMATE OF $C_{A}$}

In order to assess the influence of heat losses to the the wall, the power $P$ is shown in Fig. 5 as function of $y$ for the case of two parallel plates considered previously. The figure is based on a single chamber, which corresponds to setting $C_{r}=0$. It follows that $\Omega_{d}=\infty$, $\alpha_{d}=1+G b, \beta_{d}=G a$, in agreement with [6]. In obtaining Fig. 5, the value of $G$ was taken equal to 1 . At large values of $y$, the results depend strongly on the value of $C_{A}$. Large values of $C_{A}$ here correspond to a large effect of turbulence, and cause significant increases in the power dissipated as compared with the laminar case $\left(C_{A}=0\right)$. The results are essentially independent of $C_{A}$ in the nearly isothermal region $(y \ll 1)$. The power dissipated goes to 0 in the isothermal limit $(y \rightarrow 0)$ as well as in the adiabatic limit $(y \rightarrow \infty)$.

The previous work by Cantelmi et al [9]. has shown that heat losses to the walls in Stirling engines correspond to $C_{A}$ values of about 0.3 to 0.6. As part of the present work, experiments were carried out to determine a value of $C_{A}$ appropriate for chamber dimensions and a geometry characteristic of pulse tubes. The experimental set-up used was similar to that sketched in Fig. 1, except that there was no reservoir. The regenerator was simulated by an adjustable valve. The piston was connected to a linear motor. Measurements were made of the current delivered to the motor as a function of time under zero-load conditions. This current is proportional to the force on the piston. At sufficiently small amplitudes of the piston motion, the spring force exerted by the flexure bearings is negligible. The force delivered to the piston then serves only to overcome the inertial force $m d^{2} x / d t^{2}$. The mass $\mathrm{m}$ of the piston is known $(\mathrm{m}=0.165 \mathrm{~kg})$. The electronics provided with the motor yielded the value of the amplitude $\Delta x$. This allows determination of the inertial force, and hence of the constant of proportionality between current and force. The resulting value was $15.9 \mathrm{~N} / \mathrm{A}$. The electronics provided with the motor also allow determination of the phase angle $\delta_{F}-\delta_{x}$ between the force $\tilde{F}(t)$ and the motion $\mathrm{x}(\mathrm{t})$ of the piston. The pressure at which the measurements were taken was $P_{o}=1.5 \mathrm{Mpa}$. The volumes of the drive chamber and the reservoir were $V_{d}=17.5 \times 10^{-6} \mathrm{~m}^{3}$ and $V_{o}=44 \times 10^{-6} \mathrm{~m}^{3}$, respectively. The cross sectional area of the piston was $A=3.8 \times 10^{-4} \mathrm{~m}^{2}$, the piston amplitude was $\Delta x=1$ mm. Experimental results for the force amplitude $\Delta \tilde{F}$ are shown in Fig. 6, together with corresponding theoretical curves obtained with $C_{A}=7$. It is seen that the theoretical curves generally lie below the experimental data, even though the value used for $C_{A}$ is very large. While not shown here, the corresponding theoretical values for the phase angles $\delta_{F}-\delta_{x}$ are higher by about 40 degrees than the experimental data. It appears that the theory described can provide an estimate of the order of magnitude of the heat losses to the wall, but does not yield accurate quantitative results. It may be that the heat losses are dominated by convective effects, which have not been taken into account here.

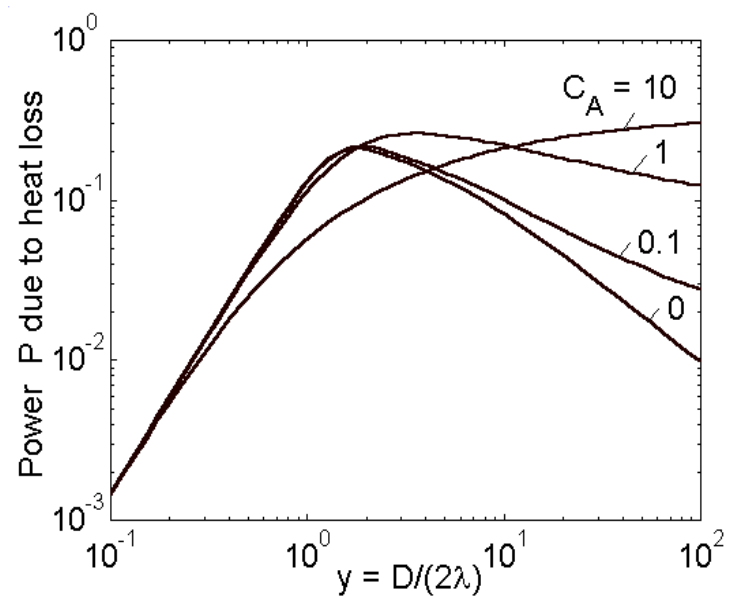

Figure 5: Power $P$ due to heat loss as function of $y$ 


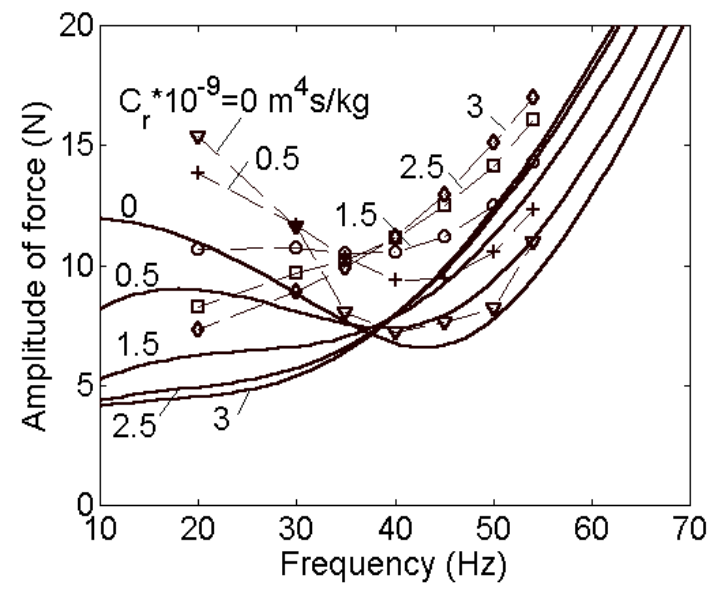

Figure 6: Amplitude of force on piston as function of frequency $\omega$

\section{APPLICATION TO ORIFICE PULSE TUBE}

The amplitude $\Delta F$ of the force on the piston for the case of a complete pulse tube is plotted in Fig. 7. The values used for geometric quantities are the same as those used in obtaining Fig. 6. Additional values used are $V_{b} / V p=10, C O_{r}=0.1$, and $C_{A}=1$. The latter value represents a level of turbulence considerably less than than that corresponding to the measurements reported in the previous section. The value $C O_{r}=0.1$ represents a regenerator with a low conductance, offering the best prospects for making use of the resonance in the drive chamber (cf. Fig. 2). In carrying out the computations, use was made of the identity

$$
\Omega_{b}=\frac{V_{b}}{V_{p}} \frac{\Omega_{p}}{O C_{o}},
$$

which follows from Eqs. (16) and (22). The value used for $O C_{o}$ is given by

$$
O C_{o, \Delta x}=\Omega_{p} \sqrt{\frac{(1+v)^{2}+\Omega_{p}^{2}}{v^{2}+\Omega_{p}^{2}}},
$$

where $v=\left(T_{h} / T_{c}\right)\left(V_{p} / V_{d}\right)$. As shown in [10], this expression optimizes the enthalpy flux for given amplitude $\Delta x$ of the piston.

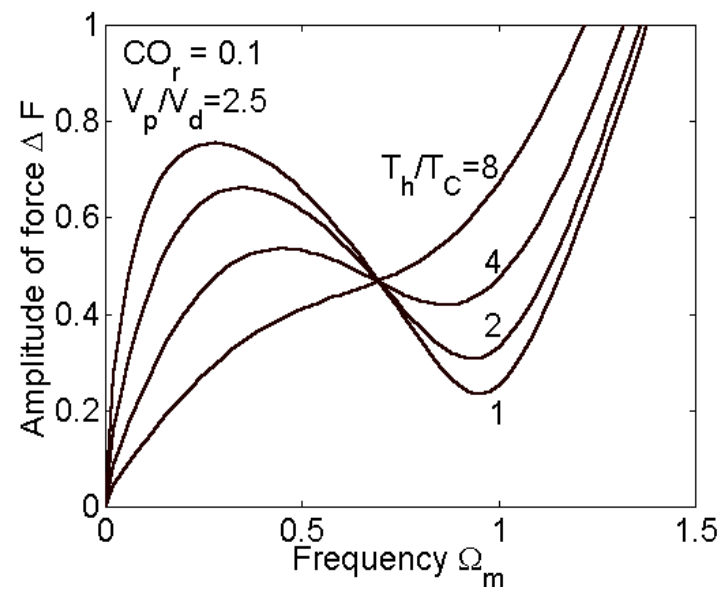

Figure 7: Amplitude of force $\Delta F$ as function of frequency $\Omega_{m}$ for pulse tube, at various values of temperature ratio $T_{h} / T_{c}$. 
Fig. 7 shows that there is a pronounced resonance for low values of $T_{h} / T_{c}$. However, this resonance disappears for values of $T_{h} / T_{c}$ that are of practical interest. Dissipation corresponding to values of $C_{A}$ larger than 1 would make the resonance disappear at still lower values of $T_{h} / T_{c}$. On the other hand, it was found that for values of $C O_{r}$ and $C_{A}$ much smaller than one there is a pronounced resonance even at high values of $T_{h} / T_{c}$. It can be concluded that in order to make beneficial use of piston resonance in pulse tubes, it is necessary to use a regenerator of low dimensionless conductance, and to minimize heat losses due to turbulence and convective effects.

\section{References}

[1] Radebaugh, R., "Development of the pulse tube refrigerator as an efficient and reliable cryocooler", The Proceedings of the Institute of Refrigeration, vol. 96, 1999-00 (ISBN 1872719 14 7) pp. 11 - 31.

[2] Zhu, S.W., Zhou, S.T., Yoshimura, N., and Matsubara, Y., "Phase shift effect of the long neck tube for the pulse tube refrigerator", Cryocoolers 9, Plenum Press, NY, (1997), pp.269-278.

[3] Gardner, D.L. and Swift, G.W., "Use of inertance in orifice pulse tube refrigerators", Cryogenics, vol. 37 (1997), pp.117-121.

[4] Zhu, S.W., WU, P.Y. and Chen, Z.Q., "Double inlet pulse tube refrigerators: an important improvement", Cryogenics, 1990, vol. 30, pp. 514-520.

[5] Martin, J.L. and Martin, C.M., "Pulse tube cryocoolers for industrial applications", paper presented at the 2001 CEC Conference. Available at www.mesoscopic.com, Cryogenics group.

[6] Mirels, H., "Effect of orifice flow and heat transfer on gas spring hysteresis", AIAA Journal vol. 32, (1994), pp. 1656-1661.

[7] Lee, K.P., "A simplistic model of cyclic heat transfer phenomena in closed spaces", Proceedings of the 18th Intersociety Enery Conversion Engineering Conference, IEEE, Boston, (1983), pp.720-723.

[8] Kornhauser, A.A. and Smith, J.L., Jr., "The effects of heat transfer on as spring performance", Proceedings of the 26th Intersociety energy conversion engineering conference, vol.5, IEEE, Boston (1991), pp. 180-185.

[9] Cantelmi, F.J., Gedeon, D., and Kornhauser, A.A., "An analytical model for turbulent compression-driven heat transfer", J. Heat Transfer, vol. 120 (1998), pp. 617-623.

[10] de Boer, P.C.T., "Optimization of the orifice pulse tube," Cryogenics, vol. 40, (2001), pp. 701-711. 